the following is the most interesting, because absolute recovery eventually ensued. A boy of four years had had whooping-cough for three weeks, and for this length of time had been observed to exhibit a twitching of the eyes, inability to walk, and a tendency to drop things held in his hands. The chief characteristic of the case was the trembling of the limbs and trunk, which bore a close resemblance to that of disseminated sclerosis. While at rest his body remained free from tremor, but voluntary movement produced a marked ataxia. The fundus oculi was at all times normal. Three months later he was discharged from hospital considerably improved. Two years later he was able to get along on level ground, though in a highly ataxic fashion. "His movements were precisely those of a drunken man." His limbs were strong; he was lively and active, and although he seemed in constant danger of falling he seldom fell. The arms were ataxic, but the tremor of the head and neck was scarcely noticeable. His speech was hesitating, and his voice not properly modulated. Twenty-nine years later he was seen again. He was now a man of 33 years, and a clerk by occupation. No sign of his former malady existed; he was an active man, and included hockey among his recreations.

The features of the third group, progressive cerebellar ataxy, are as follows: A child who has been perfectly healthy and of normal development until a certain age gradually develops an ataxia, which increases in severity until the patient becomes unable to get about. These cases are not, in the author's opinion, instances of Friedreich's disease, although allied to that malady; they are representatives of the group of cerebellar and spinal ataxias. The following is an example. A girl of 11 years had enjoyed good health until her ninth year, when she was observed to be losing the power of walking, while her arms were becoming weak and her speech difficult. On examination she was found to be well nourished and of fair intelligence. The upper central incisors were notched, and the spleen could be felt below the costal margin. There was no further evidence of congenital syphilis. Her articulation was slow and drawling, but all her words were easily understood. There was a marked intention-tremor of both upper and lower extremities. The child could neither stand nor walk without support, and with it was wildly ataxic. The head and trunk shared in the general intentiontremor. There was no optic neuritis, or atrophy, or nystagmus. The pupils reacted to light and accommodation. The knee-jerks were distinctly increased and the arm-jerks brisk. The plantar reflex was variable. The prognosis in the last of these three groups is always bad. The outlook for cases which fall under the head of congenital cerebellar ataxia and of acute ataxia is distinctly hopeful.

\section{HAMORRHAGIC PANCREATITIS.}

A CASE of this rare condition is described by Drs. Wigg and Marten in the March issue of the "Australasian Medical Gazette." The patient was a man of 41 years, with a good record of health. He came under observation for symptoms suggesting the onset of influenza-namely, headache, pain in the loins and limbs, shivering, and loss of appetite. His temperature was $102^{\circ} \mathrm{F}$. On the following day he was better, the fever having entirely disappeared. On the fifth day he returned to his business, but two' days later became ill again, the symptoms being epigastric pain, with frequent vomiting of a green, slimy fluid. The temperature was normal, and the bowels had been slightly moved during the day. There was a slight degree of icterus, with tenderness: over the epigastrium, but neither distension nor dullness about the belly. The urine contained a trace of bile, but no albumin. The case was at this stage regarded as one of gall-stones, and was treated with hot applications, calomel, and morphine. The next day brought an aggravation of the symptoms. Vomiting and hiccough were incessant, the jaundice more marked, and constipation complete. Things remained in this condition for two days longer, when a swelling was discovered below the right costal margin. It was about the size of a closed fist, dull on percussion, and very tender. Later in the day the swelling rapidly increased and extended downwards into the region of the right flank. It was decided that an operation was necessary. At this stage the patient was extremely ill: there was some general distension of the belly, this distension being most noticeable over the right hypochondriac region, where also there was great tenderness and an impairment of percussion resonance. The abdominal walls were rigid and moved but little on respiration.

When the belly was opened its cavity was found to contain a large quantity of brownish fluid. The gall-bladder appeared to be natural. The great omentum was much thickened, deeply injected, and curled up upon itself : it was, moreover, studded by small yellow spots of the size of miliary tubercles. The falciform ligament of the liver was covered with similar spots, and a few were visible upon the surfaces of the liver. A drainage-tube was inserted and the patient returned to bed. A small piece of the affected omentum which had been removed for examination was reported to be in a condition of fat necrosis due to an acute pancreatitis.

The patient continued to vomit incessantly, while the discharge of brownish fluid from the wound was profuse. The temperature was always low, and sometimes subnormal; the pulse was thready and rapid, and the respirations shallow. The patient died of exhaustion a week after the onset of symptoms. Throughout this period the vomiting and hiccough were constant and unrelieved by any medication except the liberal use of morphine. The discharge from the wound changed its character, and became offensive; its smell was like that of a partially digested meal. This was due to the digestion of tissue in the neighbourhood of the pancreas, as was shown by post-mortem examination. The discharge from the wound caused great digestive destruction of the skin in the neighbourhood. Details of the post-mortem examination are not supplied.

In connection with the fat-necrosis, which is commonly associated with the hæmorrhagic and necrotic varieties of aclite pancreatitis, Osler states that 
Langerhans has produced fat-necrosis by the injection of pancreatic extract into the perinephric fatty tissues of dogs. Hildebrand and Dettmer have shown experimentally that the necrosis is due to certain constituents of the pancreatic juice, but not trypsin. This ferment is steapsin, and its presence. has been demonstrated in certain instances of the disease by Flexner. This authority has also produced hæmorrhagic pancreatitis by the injection of artificial gastric juice into the pancreatic duct, Opie has also produced the condition by the injection of bile into the pancreatic duct of dogs, and has shown that the penetration of bile into the pancreas may be the cause of the disease in nature.

\section{Progress in Medicine and SURGerY.}

\section{CARCINOMA.}

On the Method of the Spread and Dissemination of Carcinoma and its Influence on Treatment. Whilst we vainly wait for any definite results from the many researches into the cause and prevention of cancer, the subject of its radical removal must remain that of the most practical importance in the study of the disease. It has been generally held that carcinoma is spread by a process of embolism of the cancer-cells in the lymphatic and bloodvessels. But in the case of the breast, Handley ${ }^{1}$ has shown that this view must be modified. According to this authority the cancer-cells actually spread along the lymphatic channels by a process of continuous growth, and that this permeating growth takes place as readily in a direction contrary to the lymph stream as with it. The first difficulty in accepting this theory is the fact that if a late case of mammary carcinoma be examined, the region immediately round the primary growth contains no permeated cancerous lymphatic trunks. Isolated nodules of growth are present which suggest the position of emboli. But it appears that if an area of the tissues at a greater distance from the growth be examined microscopically, it will be found that the lymphatic trunks are all permeated with cancer-cells. Thus, in every case three distinct zones may be distinguished. First, in the centre is the primary growth; round this is a zone in which the lymphatics are devoid of cancer-cells, but in which isolated nodules of growth may be present; and a third zone, which appears normal to the naked eye, forms the true margin of the growth, and here the lymphatic channels are filled with malignant cells. This last zone may be described as the microscopic edge of the growth. To explain the freedom of the lymph channels from cancer-cells in the intermediate zone, it is stated that a process of peri-lymphatic leucocytosis is set up by the malignant cells growing in the lymph vessels, and that this causes a fibrosis which destroys them. So that a double process of peripheral extension and central destruction of the carcinoma proceed at the same time, and the whole method of growth may be compared to that of a ringworm or serpiginous ulcer. But at the same time that the cancer-cells are permeating the lymphatic vessel, they may be forced into the lymph capillaries outwards to the skin or inwards to the body wall. And when in these situations the cancercells break through the walls of the lymph space, they then form the nucleus of a cancer nodule. In confirmation of this theory of the centrifugal spread of carcinoma are the facts of the distribution of the secondary malignant nodules in the skin and of the deposits in bones. The nodules in the skin always make their first appearance close to the primary growth, and later at points successively more and more remote from it. The area within which they are found is invariably a circle of continually increasing diameter with the primary growth at its centre. And in those rare cases when nearly the whole trunk is covered by secondary nodules, the arms below the deltoid insertion, and the legs below the middle of the thighs, always remain free. In bone metastases, too, as seen in 329 cases observed during thirty years at the Middlesex Hospital, those bones are most frequently affected which lie nearest to the primary growth. Thus, the sternum, ribs, femur, spine, humerus are the bones most often invaded, and that in the order mentioned. The fact that the femur and humerus are affected more often than such bones as the scapula is explained by the intimate relationship which the deep fascia has to the former in the situation of the trochanter and the deltoid insertion. But no explanation is offered of the facts that the clavicle is seldom affected, less often indeed, than the cranial bones, or that the bodies of the vertebræ are involved rather than the spines and laminæ. But only one case in this whole series shows involvement of bones distal to the knee or elbow, and therefore it is impossible to regard bony metastases at any rate, as embolic in origin, because if this were so it would be the distal bones which would be most often the seat of the growths. As regards visceral invasion, the same method of direct permeation of lymph channels is said to take place. In this there are three stages. First the network of lymph vessels which lies in the deep fascia is involved, then the deep branches of these vessels which perforate the body wall, and lastly the serous membrane which lines the thorax and abdomen, carries the cancerous process to the viscera. If the blood were the main channel of diffusion the thoracic viscera would naturally be invaded more frequently than the abdominal. But this is not the case. ${ }^{2}$ Indeed, the abdominal viscera are more often attacked than the thoracic, and in 12 per cent. of autopsies the abdominal viscera present metastatic growths, while the thorax is quite free. This is explained on the permeation hypothesis by the fact that in the linea alba there exists a weak spot in the defence of the body cavity where the cancer-cells find a short path from the fascial lymphatics to those of the abdominal cavity. The facts brought forward are so clear that the truth of these propositions must be admitted. But at the same time it is difficult to understand why general peritoneal infection by carcinoma is not 